\title{
Adherencia y caracterización de la población de adultos mayores usuarios de audífonos atendidos en el Servicio de Otorrinolaringología del Hospital Regional de Talca
}

\section{Adherence and characterization of elderly adults users of hearing aids attended in the Otorhinolaryngology Department at the Regional Hospital of Talca}

\author{
Patricia Oyarzún D', Daniela Quilaqueo \$².
}

\begin{abstract}
RESUMEN
Introducción: En Chile se ha descrito una baja adherencia al uso de audífonos en la población de adultos mayores, sin embargo, existe escasa información acerca de la realidad actual en regiones.

Objetivo: Determinar la adherencia al uso de audífonos y caracterizar a la población de adultos mayores beneficiarios de éstos mediante GES (Garantías Explícitas en Salud) del Hospital Regional de Talca (HRT). Asimismo, determinar las principales variables que se relacionan con la adherencia en este grupo.

Material y método: Estudio descriptivo, transversal, correlacional y analítico. Se evaluó a 78 usuarios con CIRUA presencialmente, índice de Barthel y cuestionario complementario. Además, se realizó una visita domiciliaria. Para el análisis de los datos se utilizó estadística descriptiva y pruebas de proporciones. El programa estadístico empleado fue STATA versión 11.

Resultados: La adherencia fue del 75\%. La edad tuvo una correlación significativa inversa con la adherencia, las demás variables estudiadas no estarían asociadas. No existe diferencia significativa entre la adherencia arrojada en el HRT versus la visita domiciliaria.

Conclusiones: Se sugiere reformular el abordaje al grupo de beneficiarios que tienen mayor edad, enfatizando el rol evaluativo y terapéutico del fonoaudiólogo. Se proponen futuras investigaciones en otras regiones, utilizando metodologías similares e instrumentos validados.

Palabras clave: Adherencia, GES, audífonos, adulto mayor.
\end{abstract}

\section{ABSTRACT}

Introduction: In Chile, a low adherence to the use of hearing aids in the elderly population has been described, however, there is a lack of information regarding the current situation in locations outside the capital city.

\footnotetext{
1 Fonoaudióloga. Escuela de Fonoaudiología, Facultad de Salud, Universidad Santo Tomás, Sede Talca, Chile.

2 Fonoaudióloga. Escuela de Fonoaudiología, Universidad de Talca, Chile.
} 
Aim: To determine the adherence to the use of hearing aids, and to characterize the population of elderly people that benefit from the use of the same through the Explicit Guarantees in Health (GES), in the Regional Hospital of Talca (HRT). Furthermore, evaluate the main variables related to adherence in this group.

Material and method: Descriptive, cross-sectional, correlational and analytical study. 78 users were evaluated with CIRUA, Barthel Index and a complementary questionnaire in person. Furthermore, a home visit was carried out. For the data analysis, descriptive statistics and proportions tests. Statistical software used was STATA 11.

Results: The adherence described was $75 \%$. Age had a significant inverse correlation with adherence, without an association of the other variables studied. There is no significant difference between adherences in the HRT compared with home visits.

Conclusions: It is suggested that the approach to elderly beneficiaries be reformulated, emphasizing the evaluative and therapeutic role of the speech therapist. Future research is proposed in other regions of the country, using similar methodologies and instruments validated.

Key words: Adherence, GES, Hearing Aid, Elderly.

\section{INTRODUCCIÓN}

Producto del envejecimiento, ocurre un deterioro de distintos sistemas y órganos en el ser humano $0^{1,2}$. Uno de los principales sistemas afectados es la audición ${ }^{3}$, la que a partir de los sesenta años comienza a disminuir considerablemente su capacidad de detectar sonidos y discriminar el lenguaje ${ }^{4}$. A esta alteración se le denomina presbiacusia ${ }^{5}$.

Según la Organización Mundial de la Salud se define presbiacusia como "pérdida de audición relacionada con el envejecimiento". También se describe como un "trastorno de la audición asociado con diferentes tipos de disfunción del sistema auditivo, periférico o central, que acompaña al envejecimiento y que no puede explicarse por condiciones extraordinarias de ototraumatismo, genéticas o patológicas ${ }^{6}$.

Aproximadamente una tercera parte de las personas mayores de 65 años padece pérdida de audición discapacitante ${ }^{7}$. La Organización Panamericana de la Salud (OPS), señala que el $30 \%$ de los mayores de 60 años sufre de una disminución de la agudeza auditiva, sin embargo, esta cifra aumenta hasta el $60 \%$ entre las personas de 85 años $^{8}$. En Chile, de acuerdo a la Encuesta Nacional de Salud ${ }^{9}$, la prevalencia de hipoacusia en mayores de 65 años es de $76 \%$ a $79,7 \%$, la cual aumenta a $90 \%$ en los mayores de 80 años.

La presbiacusia se caracteriza por una alteración en la percepción de algunos sonidos, lo que se acentúa cuando la persona se encuentra en am- bientes acústicamente desfavorables. En relación a las características audiológicas, se evidencia un descenso de los umbrales auditivos, afectándose principalmente las frecuencias agudas desde los $2000 \mathrm{~Hz}$. Además, se produce un acortamiento del campo auditivo dinámico debido al reclutamiento y en logoaudiometría se observa alterado el umbral de máxima discriminación de la palabra en relación con los umbrales auditivos. Por último, un gran porcentaje de pacientes también refieren presencia de acúfenos y dificultad en la detección de la señal en ambientes ruidosos ${ }^{4,10}$.

Dentro de las ayudas técnicas disponibles para esta patología, se encuentran los audífonos, que en Chile son otorgados en su mayoría a través del programa GES (Garantías Explícitas de Salud) a todos los adultos mayores de 65 años que presenten hipoacusia bilateral ${ }^{11}$. Sin embargo, según estudios publicados, en Chile existiría una baja adherencia al uso de éstos ${ }^{12,13}$.

Lo anterior va en desmedro del estado de salud general y de la calidad de vida de estos pacientes, no cumpliéndose los objetivos sanitarios propuestos por el programa GES para este grupo de edad, ya que una pérdida auditiva trae consigo dificultades en la socialización al obstaculizarse esta vía sensorial importante para la comunicación oral, pudiendo conllevar a un aislamiento social y deterioro cognitivo $0^{3,14-16}$.

Existen en Chile escasos estudios que informen la situación actual en regiones sobre la adherencia 
al uso de audífonos en estos pacientes y las razones por las cuales no lo utilizan. Esto se ha transformado en un tema de interés por el impacto que ocasiona la pérdida auditiva en la salud de los adultos mayores y por otra parte a nivel de políticas públicas, ya que son altos los costos que demanda la adaptación audioprotésica sumado a las numerosas necesidades que se deben cubrir, puesto que implica recursos no sólo en dispositivos auditivos, sino que también en horas médicas y evaluaciones de los distintos profesionales involucrados ${ }^{17,18}$.

\section{OBJETIVO}

El presente estudio tiene por objetivo determinar la adherencia al uso de audífonos y caracterizar a la población de adultos mayores beneficiarios de éstos a través de programa GES atendidos en el Hospital Regional de Talca, así como también, determinar las principales variables que se relacionan con la adherencia al uso de audífonos en estos usuarios.

\section{MATERIAL Y MÉTODO}

Estudio descriptivo, transversal, correlacional y analítico, que contó con la debida aprobación del Comité de Bioética de la Universidad Andrés Bello. La muestra se obtuvo durante el periodo de diciembre de 2014 a marzo de 2015 y estuvo conformada por 78 adultos mayores. Ésta se determinó con una precisión de $5 \%$, confianza de $95 \%$ y prevalencia del $5,5 \%$ de hipoacusia de acuerdo a la información referencial de la población objetivo.

Los participantes correspondían a pacientes de 65 años 0 más atendidos en el Hospital Regional de Talca beneficiarios de audífonos a través de programa GES, con al menos seis meses de uso. Además no debían presentar diagnósticos asociados a alteraciones cognitivas. Se confirmó participación voluntaria mediante consentimiento informado.

Las variables estudiadas fueron: adherencia a través de cuestionario CIRUA (Cuestionario Internacional de Resultados de Uso de Audífonos) ${ }^{19}$, edad, sexo, escolaridad, residencia y manejo del audífono por medio de cuestionario complementario. Las comorbilidades, grado de hipoacusia y modo de adaptación se obtuvieron de la revisión de fichas clínicas de los usuarios. El grado de autovalencia se midió con índice de Barthel ${ }^{20}$.

EI CIRUA es un instrumento recomendado en la etapa de seguimiento de los usuarios de audífonos según la guía GES referente al tema, ya que cuenta con su validación al español desde el año $2002{ }^{11}$. En Chile, se realizó una validación en el Hospital San Camilo en el Valle de Aconcagua, obteniéndose resultados positivos en cuanto a la confiabilidad del instrumento, además de su efectiva validez de contenido, criterio y constructo ${ }^{21}$.

En el caso de la variable "manejo del audífono" se consideró la demostración por partes de los usuarios en relación a saber poner y sacar el audífono, encender y apagar, cambio de pilas y limpieza.

Finalmente, se efectuó una visita domiciliaria a 22 sujetos del estudio para corroborar el uso del audífono.

Se utilizó estadística descriptiva para caracterizar a la muestra seleccionada, mientras que para el análisis y evaluación de las variables se utilizó pruebas estadísticas de proporciones; y en el caso de las asociaciones pruebas de Chi2 o bien exacta de Fischer. El programa estadístico utilizado fue STATA versión 11.

\section{RESULTADOS}

\section{Adherencia}

De los 78 individuos evaluados, se obtuvo una media aritmética de 28,2 puntos en CIRUA, con una desviación estándar de 3,7 puntos. El mínimo puntaje obtenido corresponde a 19 puntos y el máximo a 35 puntos. El coeficiente de variación es de $13,4 \%$. La distribución de la variable adherencia tiene un comportamiento normal con $p$-valor $>0,05$.

Desde el punto de vista de normalidad se adoptó el criterio de separación del puntaje por percentiles (Tabla 1), encontrándose que el $25 \%$ de la muestra obtiene un puntaje igual o menor a 25 puntos, mientras que el rango intercuartílico correspondiente al $50 \%$ de la muestra, obtiene un puntaje entre 26 y 31 puntos. Finalmente, el $25 \%$ restante, obtiene un puntaje superior a 31 puntos. 
Tabla 1. Percentiles distribución normal por puntaje total CIRUA

\begin{tabular}{|cc|}
\hline Percentil & Puntaje \\
\hline $25 \%$ & 25 \\
$50 \%$ & 28 \\
$75 \%$ & 31 \\
\hline
\end{tabular}

\section{Variables}

La edad promedio de los adultos mayores estudiados es de 77,4 años, con una desviación estándar de 6,8 y coeficiente de variación de 8,88\%, lo cual indica que la muestra es homogénea y el promedio representativo (Tabla 2). La correlación entre adherencia y edad, muestra $p$-valor $<0,05$

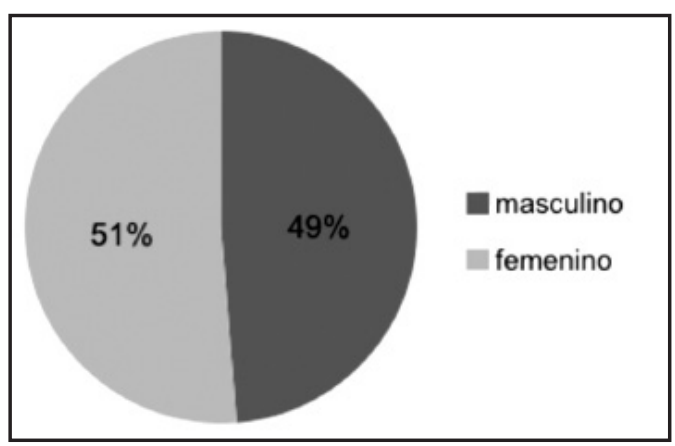

Figura 1. Distribución porcentual por sexo.

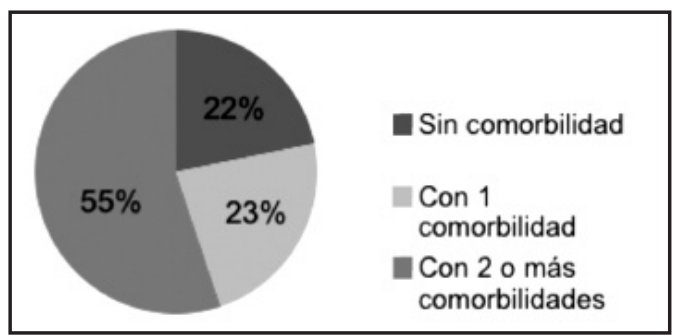

Figura 2. Distribución porcentual por comorbilidad.
$(0,014)$ con $95 \%$ de confiabilidad, existiendo una correlación significativa inversa, aunque débil, entre las variables $(-0,2772)$.

En la distribución porcentual según la variable sexo (Figura 1) se obtiene que 40 individuos son de sexo femenino y 38 son de sexo masculino. Mediante la prueba de chi cuadrado se obtiene $p$-valor $>0,05$, por tanto, no existe diferencia significativa por sexo en relación a la adherencia.

Según la distribución por comorbilidad (Figura 2), 17 individuos no presentan comorbilidad asociada, 18 presentan una comorbilidad y 43 personas manifiestan dos 0 más comorbilidades, las que principalmente fueron hipertensión, diabetes, artritis y/o déficit visual. De acuerdo a la Prueba Exacta de Fisher se obtiene $p$-valor $>0,05$ por tanto, no existe asociación significativa entre las variables adherencia y comorbilidad.

La distribución porcentual por grado de hipoacusia, en la que se consideró al oído con mayor grado de pérdida auditiva, muestra que 6 personas presentan hipoacusia de grado leve con repercusión social según HHIE-S, 45 poseen hipoacusia de grado moderado y 27 usuarios presentan hipoacusia de grado severo. No se encontró sujetos con hipoacusia de grado profundo dentro de la muestra. El valor de $\mathrm{p}$ obtenido mediante la prueba de chi cuadrado es mayor a 0,05. Por tanto, no existe asociación significativa entre las variables adherencia y grado de hipoacusia (Figura 3).

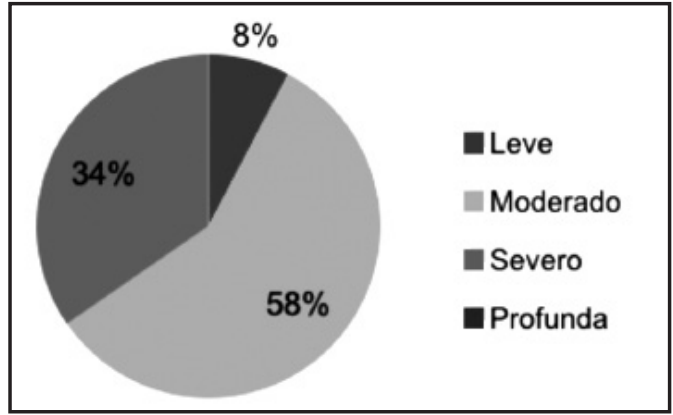

Figura 3. Distribución porcentual por grado de hipoacusia.

Tabla 2. Estadístico descriptivo por edad

\begin{tabular}{|cccccc|}
\hline N & Media & $\begin{array}{c}\text { Desviación } \\
\text { estándar }\end{array}$ & $\begin{array}{c}\text { Valor } \\
\text { Min }\end{array}$ & $\begin{array}{c}\text { Valor } \\
\text { Max }\end{array}$ & $\begin{array}{c}\text { Coeficiente } \\
\text { variación }\end{array}$ \\
\hline 78 & 77,48 & 6,88 & 66 & 94 & $8,88 \%$ \\
\hline
\end{tabular}


En relación a la distribución de la muestra por autovalencia a través del índice de Barthel (Figura 4), 1 persona señala dependencia moderada en sus actividades de la vida diaria (AVD), 11 presentan una dependencia leve y 66 personas son autovalentes. Según la Prueba Exacta de Fisher se obtiene $p$-valor $>0,05$ por tanto, no existe asociación significativa entre las variables adherencia y autovalencia.

Respecto a la distribución porcentual por escolaridad (Figura 5), muestra que 7 personas no asistieron a establecimiento educacional, 39 cursaron preparatoria, 24 cursaron humanidades, 3 pertenecen al nivel técnico y 5 cursaron educación universitaria. El valor de $\mathrm{p}$ obtenido en la Prueba Exacta de Fisher es mayor a 0,05; no existiendo asociación significativa entre las variables adherencia y escolaridad.

En los datos obtenidos en la distribución de la muestra por residencia (Figura 6), 47 residen en Talca urbano, 12 son residentes de sectores rurales de Talca y 19 personas presentan residencia en otras ciudades de la región. Mediante la Prueba Exacta de Fisher se obtiene $p$-valor $>0,05$ por tanto, no existe asociación significativa entre las variables adherencia y residencia.

Según la distribución porcentual por modo de adaptación de audífono, 50 personas presentan modo de adaptación unilateral en oído derecho, 24 tienen modo de adaptación unilateral de oído izquierdo y 4 poseen modo de adaptación bilateral (en su mayoría eran marca Widex y Audifon). De acuerdo a la Prueba Exacta de Fisher se obtiene pvalor >0,05; no existiendo asociación significativa entre las variables adherencia y modo de adaptación de audífono (Figura 7).

En relación a la distribución de la muestra por manejo del audífono (Figura 8), 10 usuarios demuestran un manejo deficiente, 8 exponen un manejo regular y 60 exhiben un buen manejo del audífono. Según la Prueba Exacta de Fisher se obtiene $p$-valor $>0,05$ por tanto, no existe asociación significativa entre las variables adherencia y manejo del audífono.

Finalmente, respecto a los datos obtenidos en la distribución de la muestra por uso de audífono en visita domiciliaria (Figura 9) demuestran que 14 personas utilizan su audífono al momento de la visita y 8 no lo utilizan.

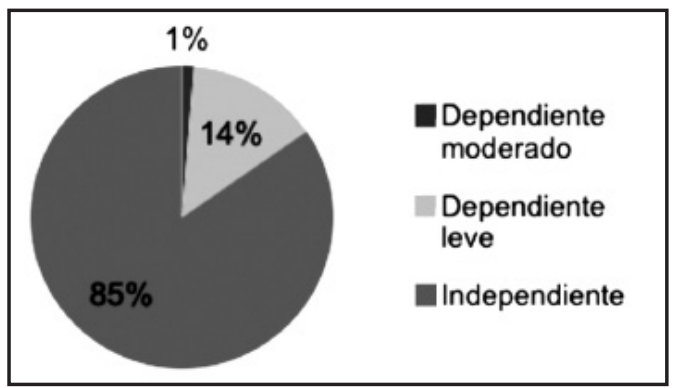

Figura 4. Distribución porcentual por autovalencia.

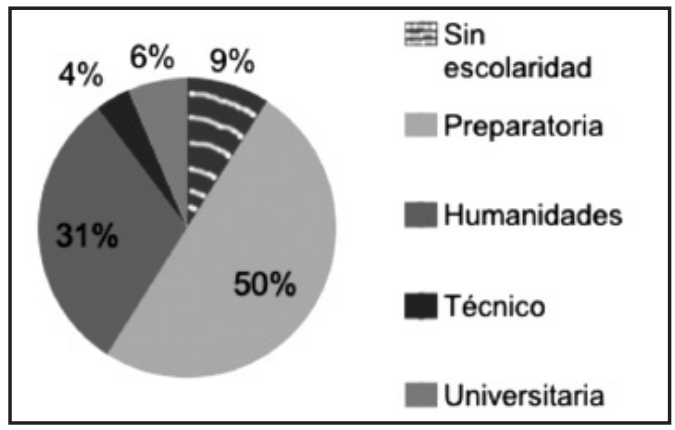

Figura 5. Distribución porcentual por escolaridad.

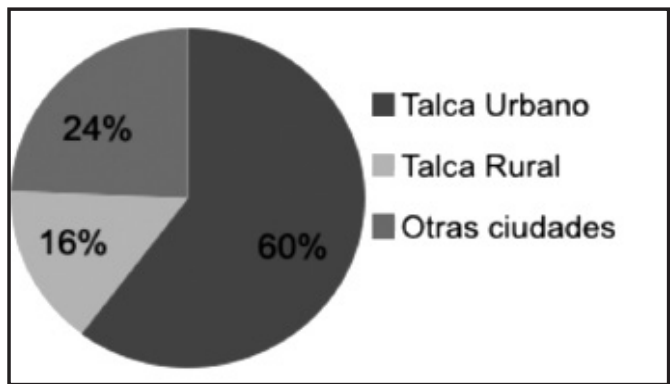

Figura 6. Distribución porcentual por residencia.

\section{DISCUSIÓN}

El $75 \%$ de adherencia, considerando satisfacción y uso del audífono, obtenido de manera presencial a través del cuestionario CIRUA en el HRT, se asemeja, aunque en menor medida, a la adherencia de $90 \%$ del estudio del Servicio de Salud de Aconcagua ${ }^{12}$, esto empleando metodologías diferentes, ya este 


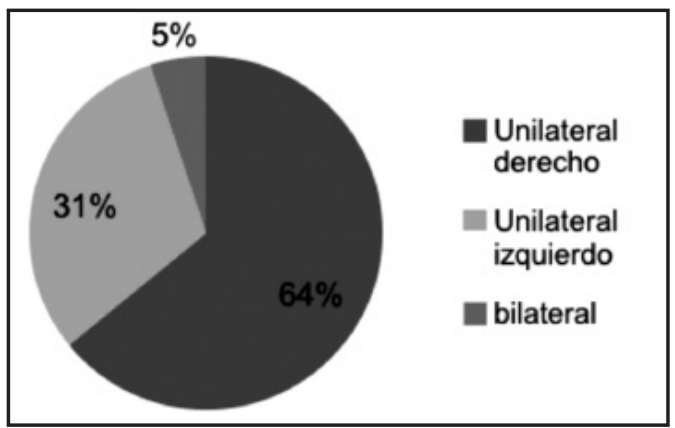

Figura 7. Distribución porcentual por modo de adaptación.

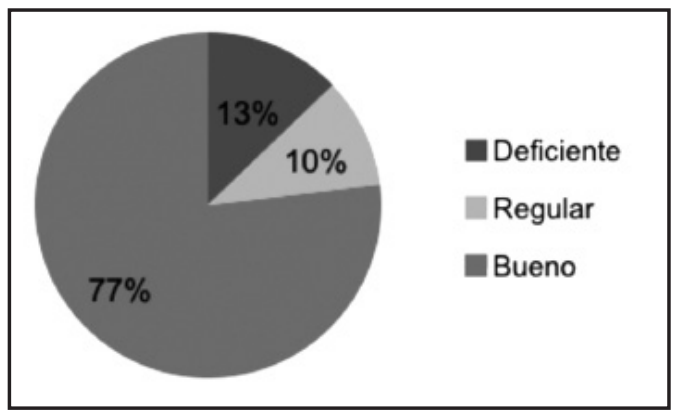

Figura 8. Distribución porcentual por manejo del audífono.

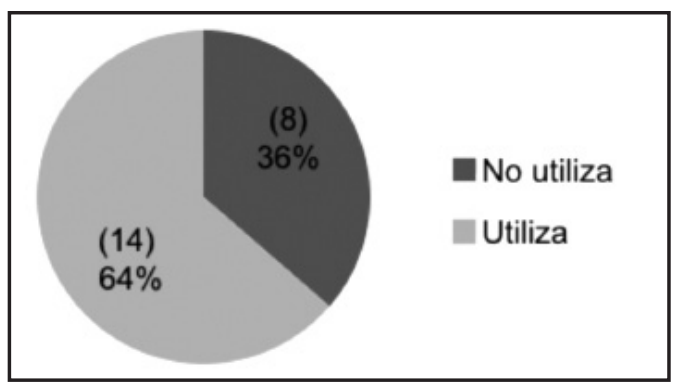

Figura 9. Distribución porcentual por uso de audífono en visita domiciliaria.

último se realizó mediante contacto telefónico y con otro tipo de cuestionario.

En relación a otras investigaciones publicadas en torno a la temática, se encontró diferencias respecto a los hallazgos del estudio de la red $\mathrm{UC}^{13}$. En este se determinó una adherencia por debajo de la nuestra, específicamente de $48 \%$, aunque al igual que el estudio anterior, fue obtenida a través de seguimiento telefónico y no de manera presencial. Esta misma modalidad se empleó en el estudio de Tamblay y cols ${ }^{14}$, pero sus resultados globales se acercan a los de esta investigación, encontrándose $80 \%$ de adherencia en la población encuestada.

En cuanto a la visita domiciliaria efectuada a una parte de la muestra en esta investigación, se comprobó que $64 \%$ utilizaba su audífono al momento de la visita. Al correlacionar estos resultados con los obtenidos en el HRT mediante el CIRUA, se determinó que no existe diferencia significativa entre tales hallazgos y que éstos son confiables. Distinto y por debajo fue lo encontrado por León y cols ${ }^{12}$ en su visita domiciliaria, en donde sólo $40 \%$ de los usuarios usaban su audífono al momento de la visita.

La única correlación significativa inversa encontrada en este estudio, fue entre las variables edad y adherencia, implicando que a mayor edad menor adherencia al uso de audífonos. La muestra estudiada tuvo una mayor distribución entre los 72 y 83 años, una media de 77,48 años y un coeficiente de variación de $8,88 \%$, revelando que la muestra es homogénea y el promedio representativo.

Lo anterior, confirma lo señalado en otras investigaciones en Finlandia ${ }^{22}$ y España ${ }^{23}$, las que mencionan a la edad como uno de los factores que influye en la baja adherencia al uso de audífonos en adultos mayores. En este sentido, los hallazgos del presente estudio vienen a complementar la información existente en la actual guía clínica GES "Hipoacusia bilateral en personas de 65 años y más que requieren el uso de audífonos"11, ya que en ésta no se menciona a la edad como uno de los factores que afectaría a la adherencia al uso de audífonos.

El resto de las variables que fueron estudiadas para caracterizar a la muestra en esta investigación, tales como: sexo, escolaridad, autovalencia, comorbilidades, residencia, modo de adaptación y manejo del audífono, resultaron sin diferencias estadísticamente significativas respecto a la adherencia al uso de audífonos.

En cuanto al sexo, en la investigación de León y cols $^{12}$ si hubo diferencias, aunque sin relevancia estadística concluyente, en este el sexo femenino tuvo una menor adherencia. Los autores explican esto en base al rol protector y de cuidado familiar que ejercen las mujeres en el hogar, las que al mis- 
mo tiempo reciben muy poco apoyo en términos del cuidado de su propia salud.

Similares diferencias se pudieron observar con el estudio de León y cols. respecto a las variables: residencia, escolaridad y comorbilidades, en donde la residencia rural, baja escolaridad y mayor número de comorbilidades, se encontrarían más en usuarios no adherentes. Tales discrepancias nuevamente podrían deberse a las diferencias metodológicas ya comentadas y al análisis de los resultados de cada estudio. También influye, principalmente en las variables: residencia y comorbilidades, el hecho de evaluar a usuarios que asistían a sus controles y no por contacto telefónico. Además de presentar comorbilidades que les permitían movilizarse a sus respectivos controles.

El grado de hipoacusia, no ha sido analizado mayormente en otros estudios realizados en Chile. Pese a esto, en un estudio cualitativo de Guerra-Zuñiga y $\operatorname{cols}^{24}$, se hace referencia que a mayor severidad de la hipoacusia, no serían tantos los cambios en la percepción auditiva de los adultos mayores y que por lo mismo la adherencia sería menos probable. En la presente investigación, la severidad se concentra entre quienes tienen una hipoacusia moderada y severa, diferencia no significativa para esta investigación en cuanto a la adherencia obtenida, quizás incide el no existir en la muestra hipoacusias profundas y escasas de grado leve, datos que se relacionan directamente con los requisitos de la guía GES.

La autovalencia tampoco ha sido mayormente investigada en este contexto en Chile, sí algunos aspectos que se podrían relacionar, como el grado de postración visto en el estudio de Tamblay y cols ${ }^{14}$ en donde $13 \%$ de los usuarios no adherentes, daba como razón al no uso esta condición. Tales discrepancias podrían deberse a la distinta metodología empleada, en donde se contactó por vía telefónica, siendo posible encontrarse con esta condición; en cambio al medir la variable presencialmente, el enfoque y resultados fueron distintos. En definitiva, la no existencia de correlación en los resultados de nuestra investigación, podría deberse a que el $85 \%$ de la muestra en este estudio, son autovalentes en sus AVD y el bajo porcentaje restante que son "dependientes" tampoco presentaron una baja adherencia significativa, asistiendo a sus controles, algunos incluso con apoyo de un acompañante 0 familiar cercano.

En la variable modo de adaptación, los resultados pueden compararse con los de la red UC ${ }^{13}$, en donde tampoco hubo diferencias significativas al comparar la implementación unilateral versus bilateral. En las demás investigaciones revisadas no se considera esta variable, quizás por la baja cantidad de pacientes GES que adquieren un segundo audífono por esta vía, lo que queda en evidencia en esta investigación, ya que sólo 5\% de la muestra poseía una adaptación bilateral. Frente a esto, se recomienda que en futuras investigaciones se analicen las características de los usuarios y el desempeño auditivo entre ambos tipos de adaptación, a modo de reflejar los beneficios en la salud de quienes reciben la segunda órtesis, mientras se incrementa la adherencia y mejora la calidad de vida.

Respecto a la variable manejo del audífono, se obtuvo un mayor porcentaje de usuarios con un "buen manejo", esto se asocia con la realidad de la muestra estudiada, puesto que en su mayoría son adherentes, aquellos que refirieron un manejo regular 0 malo, en general recibían asistencia por parte de un familiar y/o cuidador, facilitándose en cierta medida el uso. Lo opuesto se encontró en el estudio de Guerra-Zúñiga y cols ${ }^{24}$, en éste se concluyó que, desde la perspectiva de los usuarios, sería el miedo a romper el audífono 0 a su manipulación incorrecta, una de las principales razones al bajo uso; a su vez desde la perspectiva del programa GES, sería la entrega masiva de la órtesis auditiva en un tiempo escaso. En base a esto, los resultados mostrados en la presente investigación denotan una realidad más satisfactoria en el HRT, ya que se realiza un mayor seguimiento y control a los usuarios, abordando no sólo el funcionamiento del audífono, sino que también la educación sobre su uso.

Se propone para el abordaje de estos pacientes realizar una intervención posentrega del audífono con el programa Active Communication Education (ACE), el cual consiste en una estrategia de consejería y educación basada en resolución de problemas. De acuerdo a estudios realizados a nivel naciona ${ }^{25}$ e internacional ${ }^{26}$, se ha visto que la intervención con este programa en pacientes usuarios de audífonos, incrementa el tiempo de uso y mejora la adherencia al uso de éstos. Por otra parte, se sugiere incluir en la intervención habilidades de procesamiento auditivo central ${ }^{27}$.

Por último, existen variables que no se evaluaron en este estudio y que pudiesen ser de 
relevancia para futuras investigaciones, como es la valoración del estado cognitivo/mental de los usuarios, la discriminación auditiva de éstos con y sin audífono, el método prescriptivo del audífono y aspectos relacionados con el ámbito terapéutico y/o de entrenamiento en el uso del audífono. De esta manera se podría evidenciar la importancia del rol del fonoaudiólogo en la intervención de las habilidades comunicativas de esta población, favoreciendo así la adherencia al uso de audífonos.

\section{CONCLUSIÓN}

La adherencia encontrada en el HRT refleja el resultado positivo con el uso de audífonos de

\section{BIBLIOGRAFÍA}

1. Marín $P, G a c H$. Manual de Geriatría y Gerontología. 2000: 16. Disponible en [http://escuela.med.puc. cl/publ/ManualGeriatria] Revisado el 14 de Junio de 2014.

2. Castanedo C, García M, Noriega M \& Quintanilla $M$. "Consideraciones generales sobre el envejecimiento". En: Política nacional de envejecimiento y vejez. 2007: 9-11. Disponible en [https://www.minsalud.gov.co/Documentos $\% 20$ y \% 20 Publicaciones/POLÍTICA\% 20 NACIONAL\%20DE\%20ENVEJECIMIENTO\%20 Y\%20VEJEZ.pdf] Revisado el 10 de Junio de 2014.

3. Fortunato S, Forli F, Guglielmi V, De Corso; Paludetti G, Berrettin $S$ \& Fetoni. A review of new insigtts on the association between hearing loss and cognitive decline in ageing. Acta Otorhinolaryngol 2016; 36: 155-66.

4. AвELló P. Presbiacusia. Exploración e intervención, Reflexiones y pautas de actuación. Barcelona: Ed. Elsevier. 2010: 7-16. Revisado el 6 Junio de 2014.

5. Cabello E, Bahamondes $\mathrm{H}$. El adulto mayor y la patología otorrinolaringológica. Rev Hosp Clín Univ Chile 2008; 19: 21-9.

6. Gil-Carcedo L, Vallejo L, \& Gil-Carcedo, E. Otología. 21 ( $2^{\circ}$ ed). Buenos Aires: Ed. Médica Panamericana. 2004; 305-9.

7. Organización Mundial de la Salud. Sordera y pérdida de la audición. 2015: 300. [Disponible estos usuarios según las dimensiones que evalúa el CIRUA.

Existe una menor adherencia a medida que aumenta la edad y viceversa, las demás variables estudiadas no mostraron diferencias estadísticamente significativas en relación a la adherencia. Estos resultados complementan la información existente en la actual guía GES, y promueven futuros cambios en el abordaje de beneficiarios de audífonos que presentan mayor edad.

\section{Agradecimientos}

Al Servicio de Otorrinolaringología del Hospital Regional de Talca.

en http://www.who.int/mediacentre/factsheets/ fs300/es/] Revisado el 20 de febrero de 2016.

8. Organización Panamericana de la Salud. Disminución de la Agudeza Auditiva. Guía de Diagnóstico y Manejo. Oficina Regional de la Organización Mundial de la Salud. SN: 227. [Disponible en http://www.minsa.gob.pe/portal/ servicios/susaludesprimero/adultomayor/ documentos/03Guias/Guia10.pdf] Revisado el 03 de Junio de 2014.

9. Ministerio de Salud. Encuesta Nacional de Salud. Santiago; Chile. 2003. [Disponible en http://www.medicinadefamiliares.cl/Protocolos/ encnacsalres.pdf] Revisado el 05 de Junio de 2014.

10. Ciges M, Fernández-Cervilla F, Cortés R. Presbiacusia. En Suárez C. Tratado de Otorrinolaringología de Cirugía de cabeza y cuello. Madrid: Ed. Proyectos Médicos. 2000; 1395-1405.

11. Ministerio de Salud. Guía Clínica Hipoacusia Bilateral en Personas de 65 años y más que requieren uso de Audífono. 2013; 8-26.

12. León A, Ediap R, Carvallo R. Adherencia al uso de audífonos en adultos mayores del Servicio de Salud Aconcagua. Rev Otorrinolaringol Cir Cabeza Cuello 2010; 70: 37-42.

13. Maul X, Rivera C, Aracena K, Slater F, Breinbauer H. Adherencia y desempeño auditivo en uso de audífonos en pacientes adultos hipoacúsicos atendidos en la Red de Salud UC. Rev 
Otorrinolaringol Cir Cabeza Cuello 2011; 71 : 225-30.

14. Tamblay N, Villalobos I, Pastene A, Rahal M. Impacto social del uso de audífonos en adultos mayores. Rev Otorrinolaringol Cir Cabeza Cuello 2008; 68; 21-6.

15. American Speech Language Hearing Association. La pérdida de audición y la edad. Serie informativa de audiología. 2016: 1-2. [Disponible en http:// www.asha.org/uploadedFiles/La-perdida-deaudicion-y-la-edad.pdf] Revisado el 22 de Agosto de 2016.

16. Valero-García, J, Ivern I. Relación entre audición y cognición durante el envejecimiento: La presbiacusia. 2016: 1-12. [Disponible en https://www.researchgate. net/publication/303400379_RELACION_ENTRE AUDICION_Y_COGNICION_DURANTE_EL_ ENVEJECIMIENTO_LA_PRESBIACUSIA] Revisado el 12 de Septiembre de 2016.

17. The Joint Committee on Public Health. Review and Evaluation of Proposed Legislation Entitled: An Act to Provide Coverage for Hearing Aids House Bill 3598. 2010: 1-23. [Disponible en http://archives. lib.state.ma.us/bitstream/handle/2452/58034/ ocn690300026. pdf? sequence $=1$ \& isAllowed=y] Revisado el 14 de Junio de 2014.

18. Benavides P, Castro R, Jones I. "Sistema Público de Salud Situación actual y proyecciones fiscales 2013-2050". 2013: 22-24. [Diponible en http:// www.dipres.gob.cl/572/articles-114714_doc_ pdf.pdf ] Revisado el 6 de Junio de 2014.

19. Cox R, Stephen D, Kramer S. Translations of the International Outcome Inventory for Hearing Aids (IOI-HA): Traducciones del Inventario Internacional de Resultados para Auxiliares Auditivos (IOI-HA). Int J Audio/2002; 41(1): 3-26.

20. Cid-Ruzafa J, Damiá-Moreno J. Valoración de la discapacidad física: El Índice de Barthel. Rev Esp Salud Pública 1997; 71: 177-137.
21. Arapob J, Arenas F, Donoso A, Valenzuela G. Validación del cuestionario internacional del resultado de uso de audífonos CIRUA en mayores de 65 años beneficiarios Ges del Hospital San Camilo en el Valle del Aconcagua (Tesis de pregrado inédita). Universidad de Valparaíso. San Felipe. 2013; 25-53.

22. Salonen J, Johansson R, Karjalainen T, Vahlberg $T$, JERO JP, IsOAHO R. Hearing aid compliance in the elderly. B-ENT 2013; 9: 23-8.

23. Andrade, J. (2015). Presbiacusia. Adherencia al uso de audífonos en personas mayores. 2015: 11-26. [Disponible en: http://ruc.udc. es/dspace/bitstream/handle/2183/14515/ AndradeGonzález_\%20Judit_TFM_2015. pdf?sequence=2] Revisado el 20 de febrero de 2016.

24. Guerra-Zúñiga M, Cardemil-Morales F, AlbertzArévalo N, Rahal-Espejo M. Explicaciones al no uso de audífonos en un grupo de adultos mayores de Santiago de Chile. Un estudio cualitativo. Acta Otorrinolaringol Esp 2014; 65: 8-14.

25. Cardemil, F, Barría, T, Aguayo, L, Esquivel, P, Rahal, M, Fuente, A, Carvajal, R Fromin, R, VILlaLobos, I. Evaluación del programa "Active Communication Education" para rehabilitación auditiva en adultos mayores con hipoacusia usuario de audífonos. Rev Otorrinolaringol Cir Cabeza Cuello 2014; 74: 93-100.

26. Hickson L, Worral L, Scarinci N. A randomized controlled trial evaluating the active communication education program for older people with hearing impairment. Ear Hear 2007; 28(2): 212-30.

27. Cardemil, F, Muñoz D, Fuentes, E. Hipoacusia asociada al envejecimiento en Chile: ¿En qué aspectos se podría avanzar? Rev Otorrinolaringol Cir Cabeza Cuello 2016; 76: 127-35. 